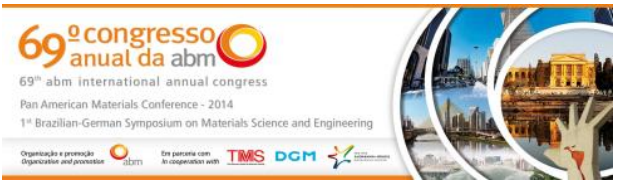

Tema: Diagrama de Fases e Transformação de Fases

\title{
EFEITOS DE TEMPERATURA DE AUSTENITIZAÇÃO, TAXA DE RESFRIAMENTO E ELEMENTOS DE LIGA NAS TRANSFORMAÇÕES DE FASES DE UM AÇO C-MN-V*
}

\author{
Charles de Abreu Martins ${ }^{1}$ \\ Nina Fonstein ${ }^{2}$ \\ Leonardo Barbosa Godefroid ${ }^{3}$ \\ Francisco José Martins Boratto ${ }^{4}$
}

\section{Resumo}

Os efeitos da temperatura de austenitização, taxa de resfriamento e adições de $\mathrm{Cr}$ ou Mo nas transformações de fases de um aço $0,12 \mathrm{C}-1,70 \mathrm{Mn}-0,2 \mathrm{~V}$, utilizando dilatometria, foi investigado. Os diagramas em resfriamento contínuo de sete aços foram elaborados e as microestruturas foram identificadas utilizando microscopias ótica e eletrônica de varredura. Em temperaturas de austenitização mais elevadas, taxas de resfriamento mais altas e maiores adições de elementos de liga ( $\mathrm{Cr}$ ou $\mathrm{Mo}$ ), houve uma elevação significativa da temperabilidade e queda na temperatura Ar3. Uma comparação entre os efeitos do $\mathrm{Cr}$ e o Mo também foi realizada, mostrando um efeito mais efetivo do Mo. Foi observada redução na temperatura $M_{s}$ com 0 decréscimo da taxa de resfriamento. Não foi observada variação significativa de $\mathrm{Ms}_{\mathrm{s}}$ com o aumento da temperatura de austenitização ou adição de elementos de liga.

Palavras-chave: Dilatometria; Transformação de fases; Temperabilidade; Efeitos de Cr e Mo.

\section{EFFECTS OF AUSTENITIZING TEMPERATURE, COOLING RATE AND ALLOYING ELEMENTS ON PHASE TRANSFORMATIONS IN C-MN-V STEEL \\ Abstract}

The effects of austenitizing temperature, cooling rate and Mo or $\mathrm{Cr}$ additions on phase transformations in a $0.12 \mathrm{C}-1.70 \mathrm{Mn}-0.2 \mathrm{~V}$ steel using dilatometry were investigated. Continuous cooling transformation diagrams of seven steels were elaborated and microstructures were identified using optical and scanning electron microscopes. Higher austenitizing temperature, faster cooling rate or bigger alloying element additions ( $\mathrm{Cr}$ or Mo) significantly increased hardenability of austenite and reduced $\mathrm{Ar}_{3}$ temperature. A comparison between $\mathrm{Cr}$ and Mo effects was also performed showing more effective influence of Mo. It was observed reduction of $\mathrm{M}_{\mathrm{s}}$ temperature with decreasing cooling rate. It was not observed significant variation in $M_{s}$ value by increasing austenitizing temperature or alloying elements.

Keywords: Dilatometry; Phase transformation; Hardenability; Effects of Mo and Cr.

1 Engenheiro Metalúrgico, Doutor, Gerente de Metalurgia, ArcelorMittal Tubarão, Vitória, ES, Brasil.

2 Engenheira Metalúrgica, Doutora, Scientific Advisor, ArcelorMittal Global R\&D, Chicago, IN, Estados Unidos.

3 Engenheiro Metalúrgico, Doutor, Professor, Redemat, UFOP, Ouro Preto, MG, Brasil.

4 Físico, Doutor, LBT Consultoria, Belo Horizonte, MG, Brasil.

* Contribuição técnica ao 69ำ Congresso Anual da ABM - Internacional e ao 14ํㅡㄹ ENEMET - Encontro Nacional de Estudantes de Engenharia Metalúrgica, de Materiais e de Minas, 21 a 25 de julho de 2014, São Paulo, SP, Brasil. 


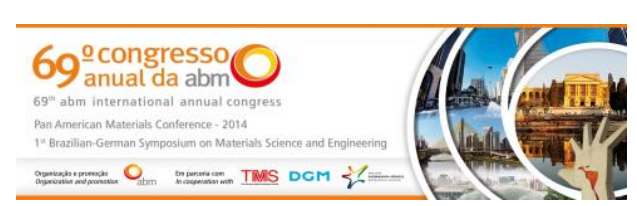

Tabela 1. Composição química dos aços investigados (\% em peso).

\begin{tabular}{ccccccc} 
Aço & $\mathrm{C}$ & $\mathrm{Mn}$ & $\mathrm{Si}$ & $\mathrm{V}$ & $\mathrm{Cr}$ & $\mathrm{Mo}$ \\
\hline Base & 0,12 & 1,69 & 0,31 & 0,20 & - & - \\
$0,25 \% \mathrm{Cr}$ & 0,12 & 1,69 & 0,30 & 0,19 & 0,25 & - \\
$0,50 \% \mathrm{Cr}$ & 0,12 & 1,70 & 0,31 & 0,21 & 0,50 & - \\
$0,75 \% \mathrm{Cr}$ & 0,12 & 1,69 & 0,30 & 0,19 & 0,76 & - \\
$1,00 \% \mathrm{Cr}$ & 0,12 & 1,70 & 0,32 & 0,20 & 1,00 & - \\
$0,25 \% \mathrm{Mo}$ & 0,12 & 1,70 & 0,31 & 0,20 & - & 0,25 \\
$0,50 \% \mathrm{Mo}$ & 0,12 & 1,69 & 0,31 & 0,20 & - & 0,50
\end{tabular}

Os ciclos térmicos estudados utilizaram duas temperaturas de austenitização: $900^{\circ} \mathrm{C}$ e $1100^{\circ} \mathrm{C}$; e sete diferentes taxas de resfriamento $\left({ }^{\circ} \mathrm{C} / \mathrm{s}\right): 0,5 ; 1,0 ; 3,0 ; 9,0 ; 20,0 ; 40,0$ e 60,0, conforme apresentado esquematicamente na Figura 1.

(a)

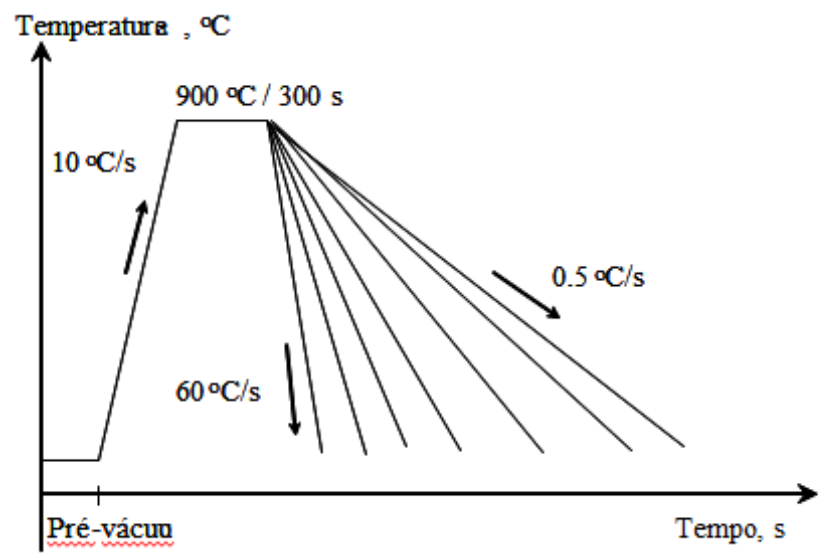

(b)

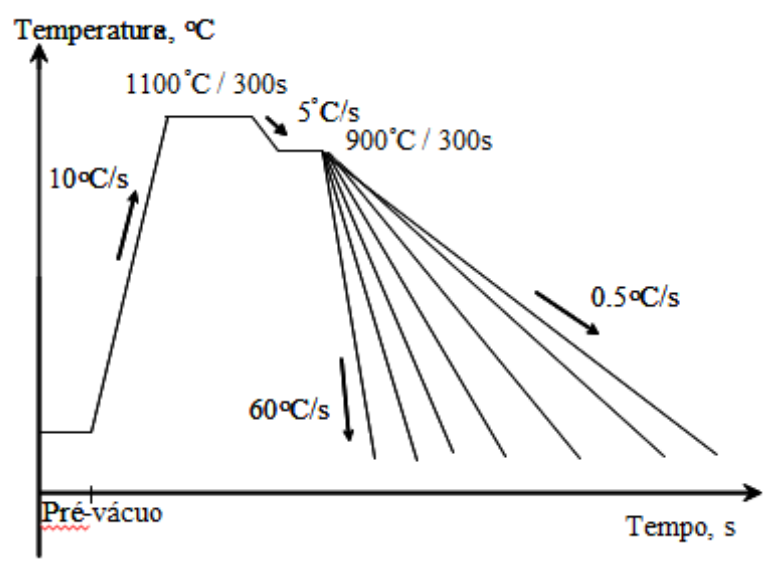

Figura 1. llustração esquemática dos ciclos térmicos adotados: (a): Ciclo $900^{\circ} \mathrm{C}$; (b) Ciclo $1100^{\circ} \mathrm{C}$.

O início e final da decomposição da austenita foram detectados pela identificação das temperaturas nas quais a curva temperatura-dilatação desviou-se da linearidade. 0 tamanho de grão austenítico prévio foi identificado utilizando-se ataque com ácido pícrico (2g), com cloreto de ferro (92g) e água destilada (100ml). O tempo de ataque foi de 1 minuto. A investigação das microestruturas finais e a quantificação das mesmas foram realizadas utilizando as microscopias ótica e eletrônica de varredura. Para tal, as amostras foram atacadas com Nital $2 \%$, utilizando-se procedimentos metalográficos padrão. As frações volumétricas dos constituintes foram calculadas manualmente utilizando-se malha com 910 pontos, com contagem em 5 campos por amostra. Adicionalmente, realizou-se microdureza com carga de $400 \mathrm{~g}$.

\footnotetext{
* Contribuição técnica ao 69ำ Congresso Anual da ABM - Internacional e ao 14ํㅡㄹ ENEMET - Encontro Nacional de Estudantes de Engenharia Metalúrgica, de Materiais e de Minas, 21 a 25 de julho de 2014, São Paulo, SP, Brasil.
} 


\section{RESULTADOS E DISCUSSÃO}

\subsection{Efeitos da Temperatura de Austenitização}

As diferentes temperaturas de austenitização utilizadas $\left(900^{\circ} \mathrm{C}\right.$ e $\left.1100^{\circ} \mathrm{C}\right)$ objetivaram gerar diferentes tamanhos de grãos austeníticos prévios ao resfriamento contínuo. Enquanto as amostras aquecidas a $900^{\circ} \mathrm{C}$ apresentaram tamanho de grão austenítico médio de $21,31 \mu \mathrm{m}( \pm 3,2 \mu \mathrm{m})$, o aquecimento a $1100^{\circ} \mathrm{C}$ gerou um valor médio $187,11 \mu \mathrm{m}( \pm 6,9 \mu \mathrm{m})$, sendo o erro com intervalo de confiança $95 \%$. Não foi observada diferença significativa de tamanho de grão austenítico em função da composição química dos aços.

A diferença significativa dos tamanhos de grãos austeníticos prévios ao resfriamento contínuo observada entre os aquecimentos a $900^{\circ} \mathrm{C}$ e $1100^{\circ} \mathrm{C}$ afetou as transformações de fase para todos os aços avaliados. A Figura 2 apresenta os diagramas de transformação em resfriamento contínuo (TRC) dos aços C-Mn-V; $0,50 \% \mathrm{Cr}$ e $0,50 \%$ Mo para diferentes tamanhos de grãos austeníticos, incluindo os valores de microdureza $(\mathrm{HV})$ para cada taxa de resfriamento utilizada.

Como pode ser visto na Figura 2, não foi observada a presença de perlita em nenhum, aço estudado, incluindo o C-Mn-V. Isso pode ser explicado pelas presenças de C, Mn e V, que elevaram a temperabilidade dos mesmos.

Após a identificação e quantificação dos constituintes presentes em cada aço, elaborou-se os gráficos apresentados na Figura 3, relativos aos aços C-Mn-V; $0,50 \% \mathrm{Cr}$ e $0,50 \% \mathrm{Mo}$, onde pode-se observar que a menor temperatura de austenitização $\left(900^{\circ} \mathrm{C}\right)$ resultou em menor presença de constituintes aciculares, principalmente bainita, para menores taxas de resfriamento. Para altas taxas de resfriamento, observa-se uma redução no volume de martensita, em relação ao ciclo $1100^{\circ} \mathrm{C}$. Esse comportamento foi observado em todos os aços estudados.

Assim, considerando a redução da temperatura de austenitização de $1100^{\circ} \mathrm{C}$ para $900^{\circ} \mathrm{C}$, os resultados apresentados na Figura 3 podem ser sumarizados conforme a seguir:

- Baixas taxas de resfriamento (menores que $20 \mathrm{C} / \mathrm{s}$ ): aumento da fração volumétrica de ferrita pelo aumento da densidade de pontos de nucleação da ferrita; aumento da fração volumétrica de martensita elo enriquecimento em $C$ da austenita remanescente, causado pela intensa transformação ferrítica; redução da transformação bainítica como consequência das condições mais favoráveis para a transformação martensítica $[9,10,15]$;

- Altas taxas de resfriamento $\left(20^{\circ} \mathrm{C} / \mathrm{s}\right.$ ou maior): a transformação ferrítica é quase completamente eliminada. Assim, o aumento da taxa de resfriamento aumenta a fração volumétrica de bainita, intensificado pelo aumento da densidade de sítios para sua nucleação nos contornos de grão da austenita, em função de suas características difusionais, reduzindo a fração volumétrica de martensita $[9,15]$.

A Figura 4 mostra que a redução da temperatura de austenitização elevou significativamente a $\mathrm{Ar}_{3}$ para todas as taxas de resfriamento, visto que o menor tamanho de grão austenítico prévio elevou a densidade de pontos para a nucleação da ferrita [16-18]. Essas elevações variaram de $29^{\circ} \mathrm{C}$ até valores mais altos que $100^{\circ} \mathrm{C}$, principalmente para taxas de resfriamento mais altas como no aço C-Mn-V em taxa de $20^{\circ} \mathrm{C} / \mathrm{s}$ (aumento de $138^{\circ} \mathrm{C}$ ) e no aço $0,50 \% \mathrm{Cr}$ em taxa de $9^{\circ} \mathrm{C} / \mathrm{s}$ (aumento de $100^{\circ} \mathrm{C}$ ). Como apresentado na Figura 4, não foi observado efeito da temperatura de austenitização no valor de $\mathrm{Ms}_{\mathrm{s}}$.

\footnotetext{
* Contribuição técnica ao $69^{\circ}$ Congresso Anual da ABM - Internacional e ao 14ํㅡㄹ ENEMET - Encontro Nacional de Estudantes de Engenharia Metalúrgica, de Materiais e de Minas, 21 a 25 de julho de 2014, São Paulo, SP, Brasil.
} 

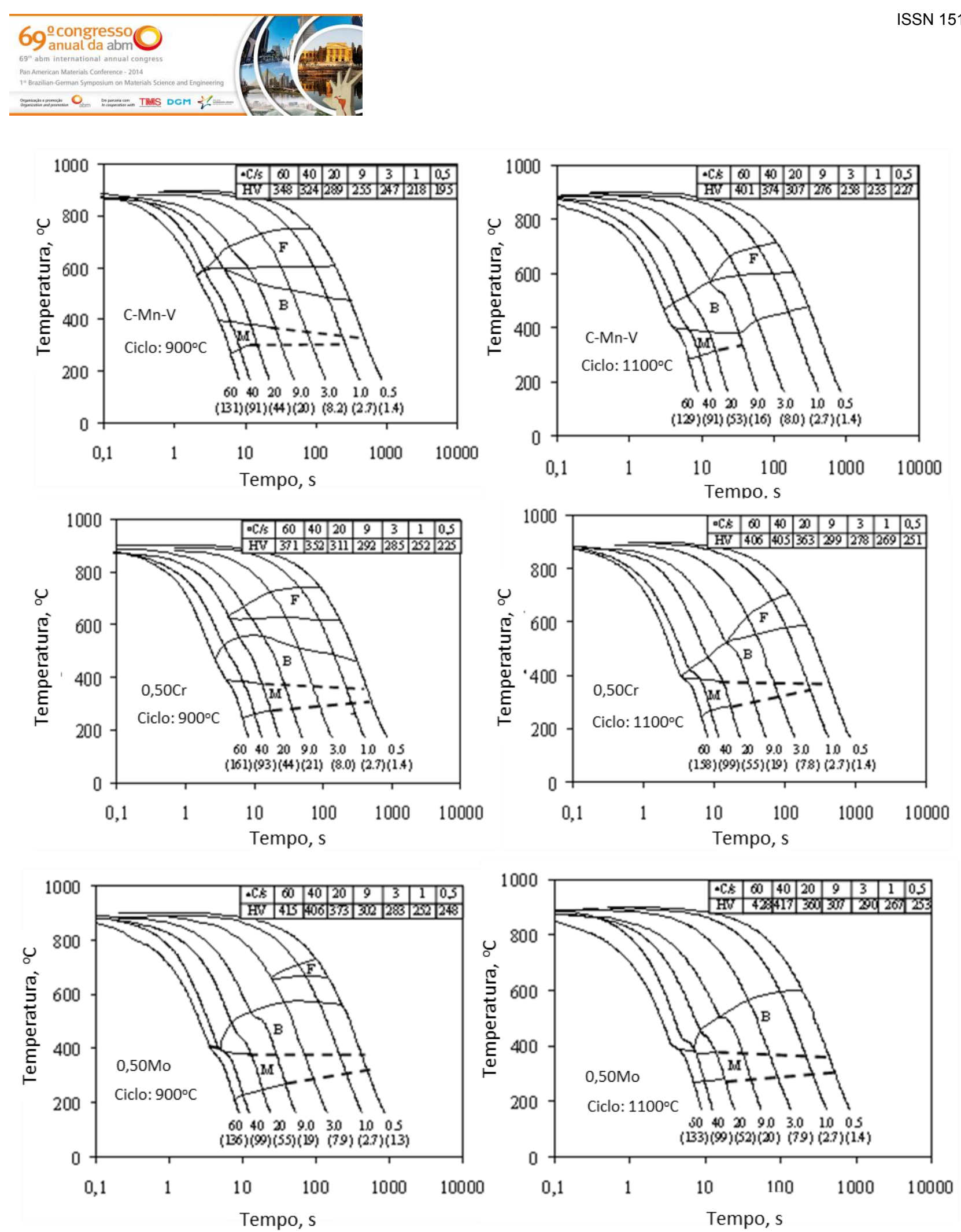

Figura 2. Diagramas em resfriamento contínuo dos aços $\mathrm{C}-\mathrm{Mn}-\mathrm{V} ; 0,50 \% \mathrm{Cr}$ e $0,50 \% \mathrm{Mo}$, com os valores de microdureza $(\mathrm{HV})$. A taxa de resfriamento média $\left({ }^{\circ} \mathrm{C} / \mathrm{s}\right)$ entre $500^{\circ} \mathrm{C}$ e $800^{\circ} \mathrm{C}$ está entre parêntesis.

\footnotetext{
* Contribuição técnica ao 69 Congresso Anual da ABM - Internacional e ao 14ํㅡㄹ ENEMET - Encontro Nacional de Estudantes de Engenharia Metalúrgica, de Materiais e de Minas, 21 a 25 de julho de 2014, São Paulo, SP, Brasil.
} 


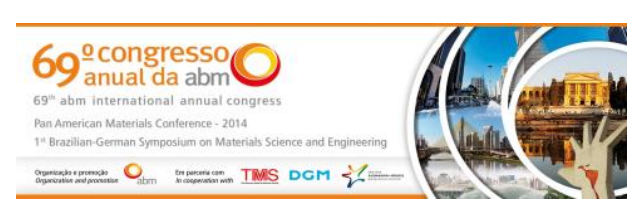

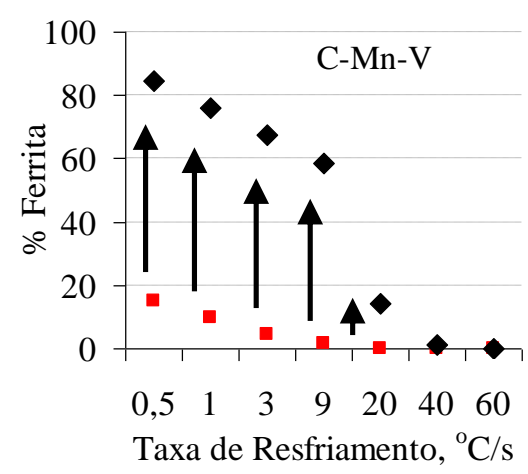
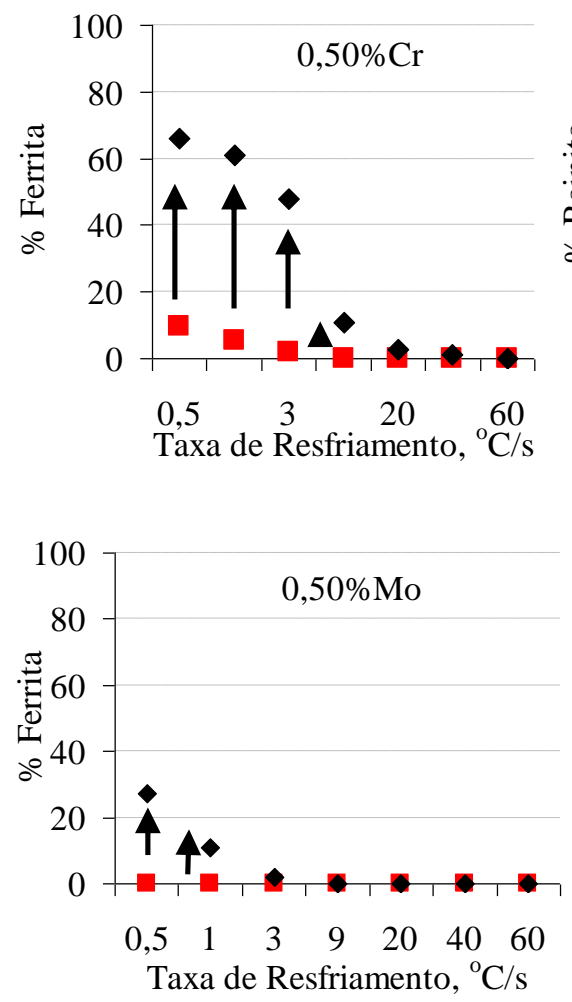
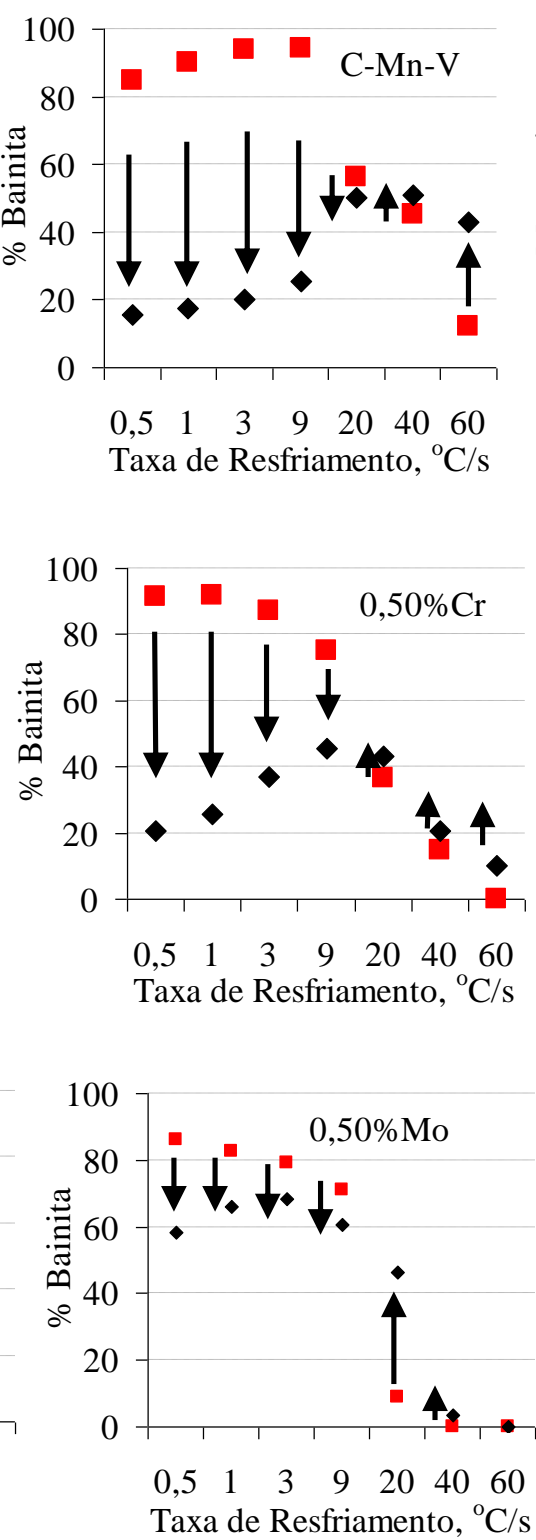


Ciclo $900^{\circ} \mathrm{C}$ Ciclo $1100^{\circ} \mathrm{C}$

Figura 3. Evolução estrutural dos aços C-Mn-V; $0,50 \% \mathrm{Cr}$ e 0,50\% Mo com a taxa de resfriamento para os dois ciclos térmicos adotados.

\subsection{Efeitos da Taxa de Resfriamento}

A variação da taxa de resfriamento afetou significativamente o valor de $\mathrm{Ar}_{3} \mathrm{em}$ todos os aços, como pode ser observado na Figura 4, aonde o valor da Ar3 reduziu com o aumento da taxa de resfriamento. Esta redução atingiu o valor máximo para o aço CMn-V: $203^{\circ} \mathrm{C}$ de $0,5^{\circ} \mathrm{C} / \mathrm{s}$ para $60^{\circ} \mathrm{C} / \mathrm{s}$ (ciclo $1100^{\circ} \mathrm{C}$ ).

Por outro lado, o aumento da taxa de resfriamento causou aumento em $\mathrm{Ms}_{\mathrm{s}}$, provavelmente porque taxas de resfriamento maiores causaram menor enriquecimento da austenita em $\mathrm{C}$ durante as transformações difusionais tais como a ferrítica, tornando a transformação displaciva mais fácil [18-20]. Esta elevação de $M_{s}$ atingiu $30,6^{\circ} \mathrm{C}$ no aço $\mathrm{C}-\mathrm{Mn}-\mathrm{V}$ (ciclo $900^{\circ} \mathrm{C}$ ).

\footnotetext{
* Contribuição técnica ao $69^{\circ}$ Congresso Anual da ABM - Internacional e ao 14ํㅡㄹ ENEMET - Encontro Nacional de Estudantes de Engenharia Metalúrgica, de Materiais e de Minas, 21 a 25 de julho de 2014, São Paulo, SP, Brasil.
} 

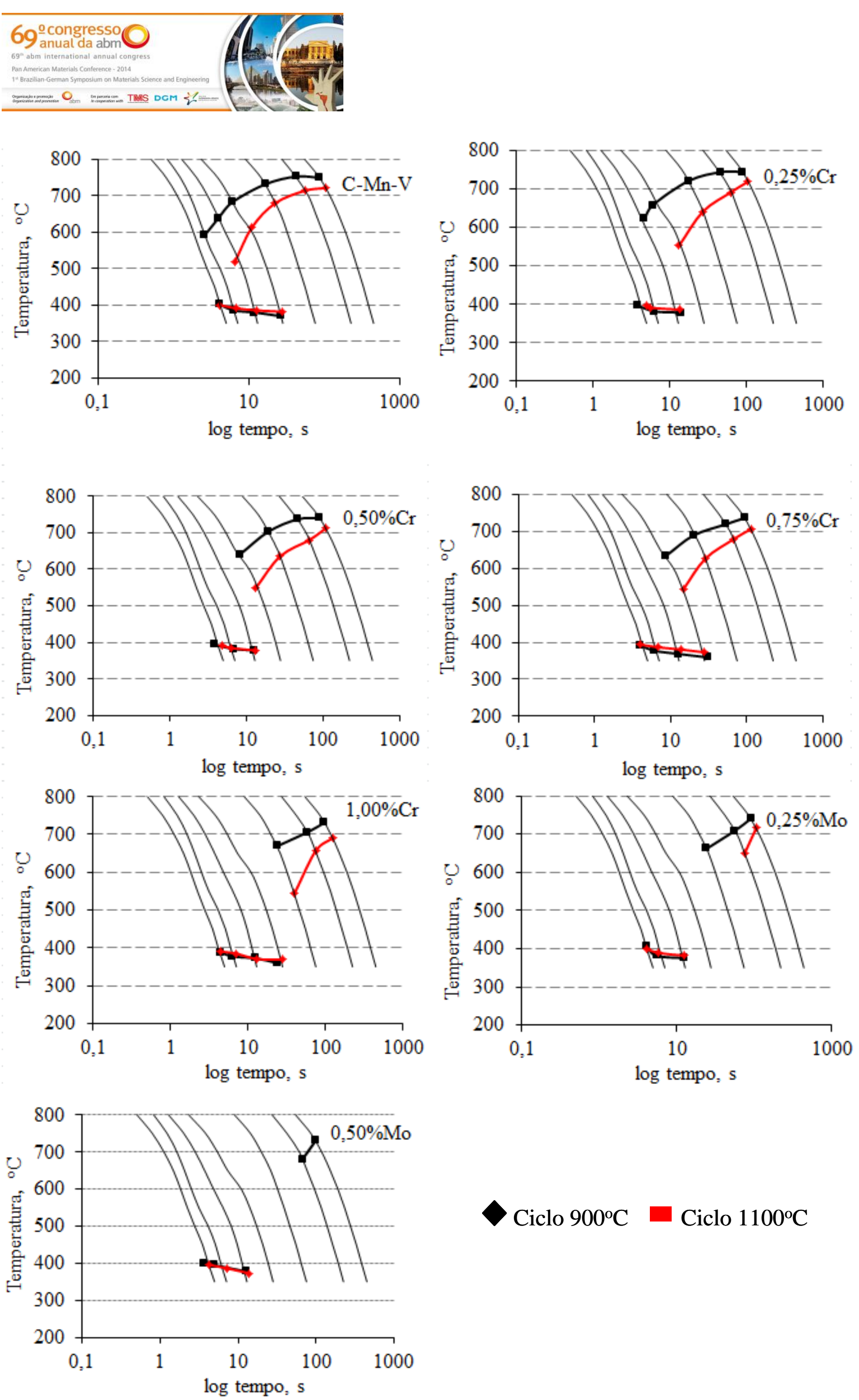

Ciclo $900^{\circ} \mathrm{C} \quad$ Ciclo $1100^{\circ} \mathrm{C}$

Figura 4. Efeito da temperatura de austenitização na $A_{3}, M_{s}$ e microdureza (nas tabelas).

\footnotetext{
* Contribuição técnica ao 69 Congresso Anual da ABM - Internacional e ao 14ํㅡㄹ ENEMET - Encontro Nacional de Estudantes de Engenharia Metalúrgica, de Materiais e de Minas, 21 a 25 de julho de 2014, São Paulo, SP, Brasil.
} 


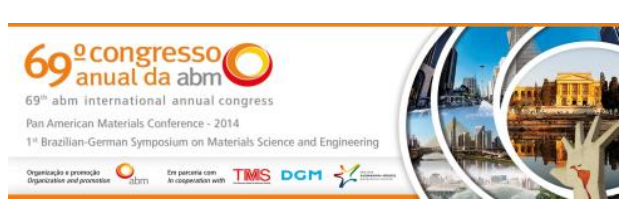

Em relação à estrutura final, na Figura 3 pode-se observar que para tamanhos de grãos austeníticos prévios maiores (ciclo $1100^{\circ} \mathrm{C}$ ), o aumento da taxa de resfriamento aumentou a fração volumétrica de martensita, reduzindo as presenças de ferrita e bainita. Como mostrado na Figura 4, o aumento da taxa de resfriamento reduziu a $\mathrm{Ar}_{3}$, suprimindo a formação da ferrita. Além disso, a redução do tempo disponível para transformações de fases difusionais promoveram a transformação displaciva da martensita. Este mecanismo torna-se facilitado no ciclo $1100^{\circ} \mathrm{C}$, por gerar austenita com maior tamanho de grão, com menor densidade de pontos de nucleação para transformações de fases difusionais, comparativamente aos tamanhos de grãos austeníticos menores obtidos no ciclo $900^{\circ} \mathrm{C}$.

Para o ciclo $900^{\circ} \mathrm{C}$, em altas taxas de resfriamento, principalmente a partir de $20^{\circ} \mathrm{C} / \mathrm{s}$, também há a redução das presenças da ferrita e bainita, aumentando a fração volumétrica de martensita. Mas neste ciclo, para taxas de resfriamento menores, a transformação martensítica competiu com a formação da bainita, enquanto a presença da ferrita foi reduzida. Para este ciclo, como o tamanho de grão austenítico prévio ficou menor que aquele obtido no ciclo $1100^{\circ} \mathrm{C}$, para taxas de resfriamento menores a maior densidade de sítios para transformação de fases difusionais favoreceram condições para a transformação bainítica.

Como apresentado na Figura 4, a elevação da taxa de resfriamento em ambos os ciclos e para todos os aços estudados causaram elevação da microdureza, devido à maior dureza da martensita, em comparação com a ferrita e a bainita.

\subsection{Efeitos dos Elementos de Liga}

Adições de Cr ou Mo elevaram a temperabilidade e reduziram os valores de $\mathrm{Ar}_{3}$, assim como afetaram as frações volumétricas dos constituintes.

A Figura 5 mostra a queda de $\mathrm{Ar}_{3}$ com a elevação da presença de $\mathrm{Cr}$ ou Mo em ambos os ciclos para todas as taxas de resfriamento. Além disso, observa-se a redução do campo ferrítico. Pode ser notado também que a adição de $0,50 \%$ Mo eliminou o campo ferrítico no ciclo $1100^{\circ} \mathrm{C}$. Já o valor de $\mathrm{M}_{\mathrm{s}}$ não apresentou variação significativa com as adições de Cr ou Mo.
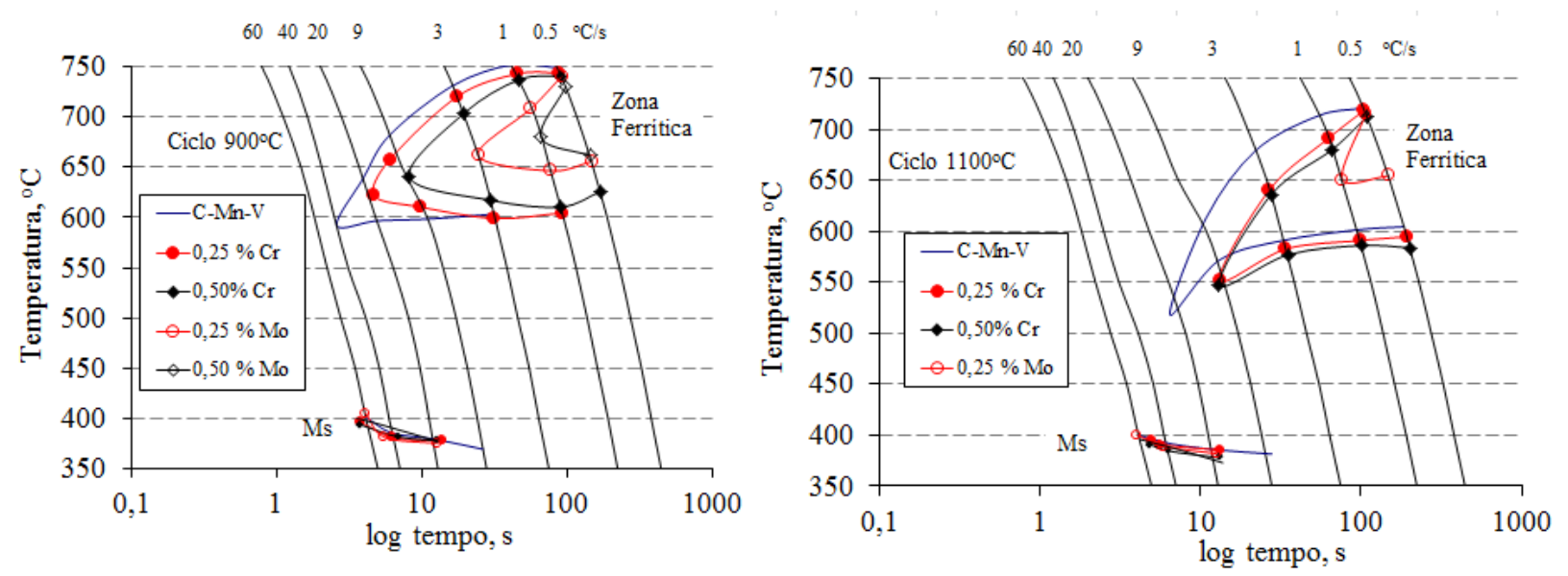

Figura 5. Efeitos de adições de $\mathrm{Cr}$ ou Mo nas temperaturas $\mathrm{Ar}_{3}$ e $\mathrm{M}_{\mathrm{s}}$ nos ciclos $900^{\circ} \mathrm{C}$ e $1100^{\circ} \mathrm{C}$ para todas as taxas de resfriamento adotadas.

\footnotetext{
* Contribuição técnica ao $69^{\circ}$ Congresso Anual da ABM - Internacional e ao 14ํㅡㄹ ENEMET - Encontro Nacional de Estudantes de Engenharia Metalúrgica, de Materiais e de Minas, 21 a 25 de julho de 2014, São Paulo, SP, Brasil.
} 
Como mostrado na Figura 6, as adições de Cr ou Mo elevaram a temperabilidade para todas as taxas de resfriamento em ambos os ciclos térmicos, aumentando a fração volumétrica de martensita.
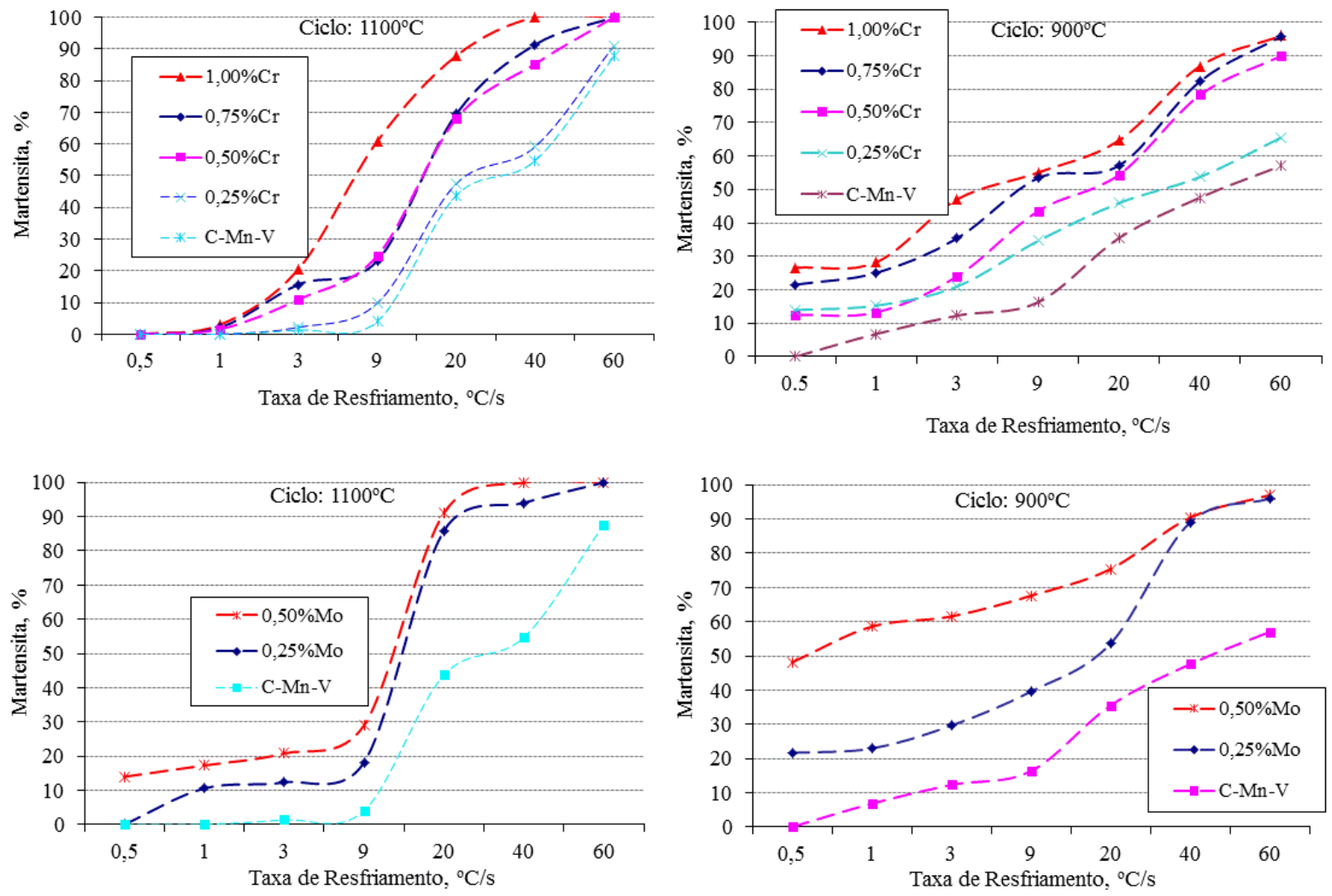

Figura 6. Efeito a adição de $\mathrm{Cr}$ ou Mo na \% de martensita nos ciclos $900^{\circ} \mathrm{C}$ e $1100^{\circ} \mathrm{C}$ para todas as taxas de resfriamento adotadas.

Quando o efeito do Cr é comparado com o do Mo, observa-se na Figura 5 que o Mo reduziu mais intensamente a $\mathrm{Ar}_{3}$ e o tamanho do campo ferrítico em ambos os ciclos. Enquanto a $0,25 \%$ Mo eliminou a transformação ferrítica a partir da taxa de resfriamento de $3^{\circ} \mathrm{C} / \mathrm{s}$, no ciclo $900^{\circ} \mathrm{C}$, e $1^{\circ} \mathrm{C} / \mathrm{s}$ no ciclo $1100^{\circ} \mathrm{C}$, para $0,25 \% \mathrm{Cr}$ esses valores foram $20^{\circ} \mathrm{C} / \mathrm{s}$ e $9^{\circ} \mathrm{C} / \mathrm{s}$, respectivamente.

Uma consequência da maior temperabilidade obtida nos aços ao Mo, em comparação aos aços ao $\mathrm{Cr}$, foi os maiores valores de microdureza, conforme apresentado na Figura 7.

\section{CONCLUSÃO}

1. Os efeitos da temperatura de austenitização, taxa de resfriamento e adição de elementos de liga nas transformações de fases e microdureza de um aço C_Mn-V foram estudadas utilizando a dilatometria e elaborando os diagramas TRC. A elevação da temperatura de austenitização de $900^{\circ} \mathrm{C}$ para $1100^{\circ} \mathrm{C}$ aumentou o tamanho de grão austenítico de um valor médio de $21,31 \mu \mathrm{m}$ para $187,11 \mu \mathrm{m}$. Como consequência, a formação da ferrita foi suprimida, a temperatura $\mathrm{Ar}_{3}$ foi reduzida $\mathrm{e}$ a temperabilidade elevada. A redução na $\mathrm{Ar}_{3}$ variou de $29^{\circ} \mathrm{C}$ a $138^{\circ} \mathrm{C}$, dependendo da taxa de resfriamento. A temperatura $M_{s}$ não sofreu variação significativa;

\footnotetext{
* Contribuição técnica ao 69ำ Congresso Anual da ABM - Internacional e ao 14ํㅡㄹ ENEMET - Encontro Nacional de Estudantes de Engenharia Metalúrgica, de Materiais e de Minas, 21 a 25 de julho de 2014, São Paulo, SP, Brasil.
} 


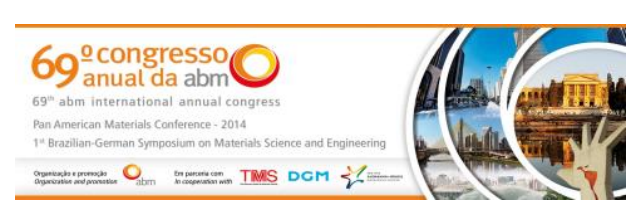

2. A redução da temperatura de austenitização de $1100^{\circ} \mathrm{C}$ para $900^{\circ} \mathrm{C}$ e a utilização de baixas taxas de resfriamento (menores que $20^{\circ} \mathrm{C} / \mathrm{s}$ ) aumentaram a fração volumétrica de ferrita e martensita, reduzindo a presença da bainita. Para altas taxas de resfriamento $\left(20^{\circ} \mathrm{C} / \mathrm{s}\right.$ ou maior), a transformação da ferrita foi quase completamente eliminada para ambas as temperaturas de austenitização e a fração volumétrica de martensita foi reduzida, aumentando a fração volumétrica de bainita;

3. Para as taxas de resfriamento utilizadas, de $0,5 \mathrm{C} / \mathrm{s}$ a $60^{\circ} \mathrm{C} / \mathrm{s}$, a elevação da mesma também aumentou a temperabilidade, aumentando a fração volumétrica de martensita e a microdureza, reduzindo as presenças de ferrita e bainita. Foi detectada uma redução da $\mathrm{Ar}_{3}$ de até $203^{\circ} \mathrm{C}$ (aço C-Mn-V, ciclo $1100^{\circ} \mathrm{C}$ ). Por outro lado, $\mathrm{M}_{\mathrm{s}}$ foi aumentada com o aumento da taxa de resfriamento, atingindo um aumento de $30,6^{\circ} \mathrm{C}$ (aço C-Mn- $\mathrm{V}$, ciclo $900^{\circ} \mathrm{C}$ );

4. As adições de $\mathrm{Cr}$ ou Mo aumentaram a temperabilidade, reduzindo a $\mathrm{Ar}_{3}$ e o campo ferrítico, sendo o efeito do Mo mais pronunciado. Enquanto a adição de 0,25\%Mo eliminou a transformação ferrítica a partir da taxa de resfriamento de $3^{\circ} \mathrm{C} / \mathrm{s}$, no ciclo $900^{\circ} \mathrm{C}$, e $1^{\circ} \mathrm{C} / \mathrm{s}$ no ciclo $1100^{\circ} \mathrm{C}$, para $0,25 \% \mathrm{Cr}$ esses valores foram $20^{\circ} \mathrm{C} / \mathrm{s}$ e $9^{\circ} \mathrm{C} / \mathrm{s}$, respectivamente.
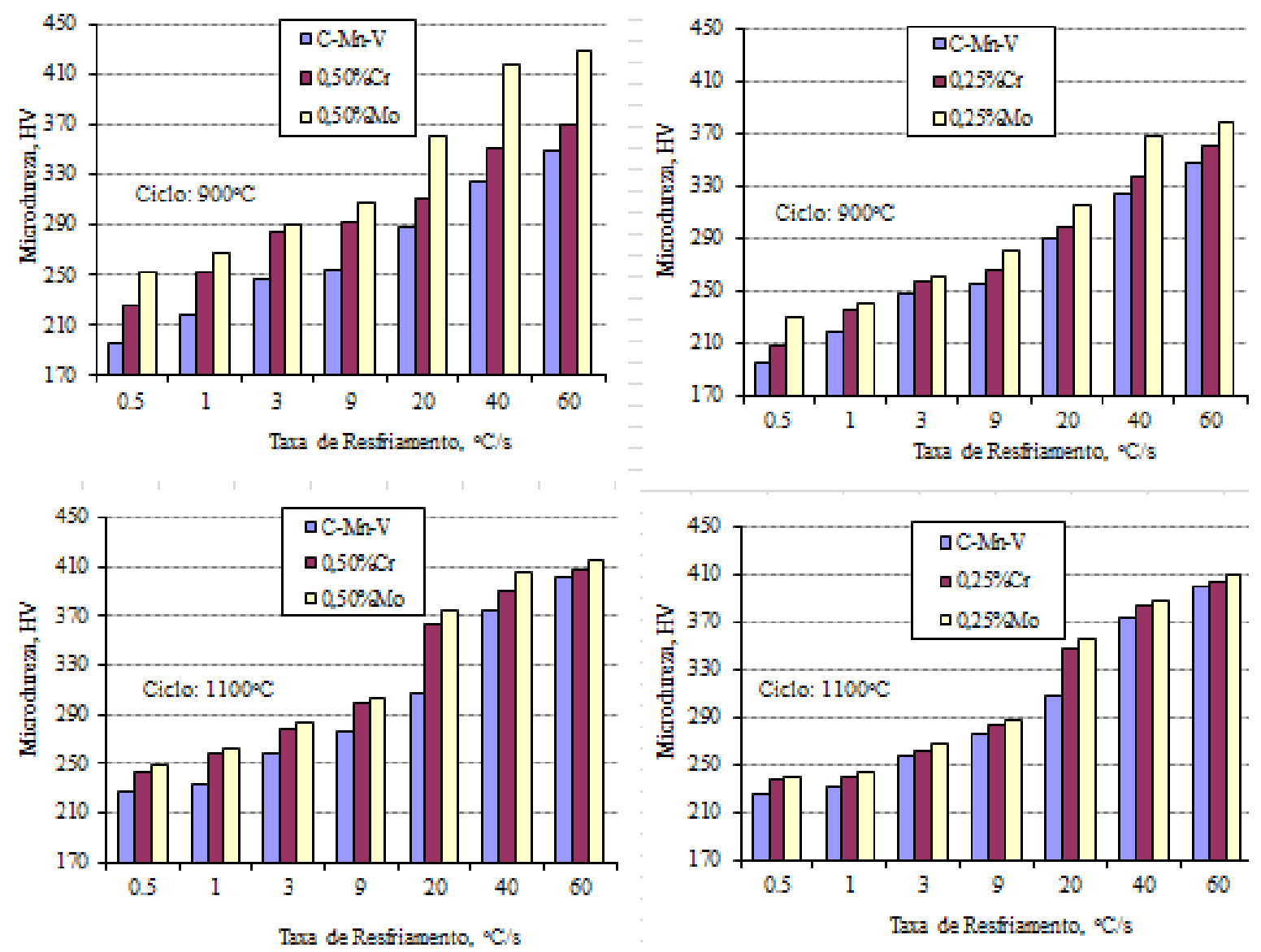

Figura 7. Efeito da adição de $\mathrm{Cr}$ ou Mo na nos ciclos $900^{\circ} \mathrm{C}$ e $1100^{\circ} \mathrm{C}$ para todas as taxas de resfriamento adotadas.

\section{Agradecimentos}

* Contribuição técnica ao 69ำ Congresso Anual da ABM - Internacional e ao 14ํㅡㄹ ENEMET - Encontro Nacional de Estudantes de Engenharia Metalúrgica, de Materiais e de Minas, 21 a 25 de julho de 2014, São Paulo, SP, Brasil. 


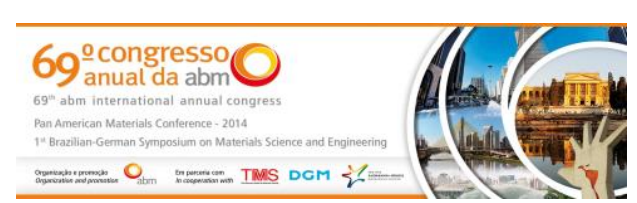

Os autores agradecem à ArcelorMittal pela autorização para a publicação deste artigo e à Dr. Astrid Perlade da ArcelorMittal Global R\&D, Maizières, França, pelas relevantes contribuições na etapa de planejamento dos experimentos.

\section{REFERÊNCIAS}

1 Yamamoto S, Yokoyama H, Yamada K, et al. Effects of the Austenite Grain Size and Deformation in the Unrecrystallized Austenite Region on Bainite Transformation Behavior and Microstructutre. ISIJ International. 1995;35(8):1020-1026.

2 Han F, Hwang B, Suh DW, et al. Effect of Molybdenum and Chromium on Hardenability of Low-Carbon Boron-Added Steels. Metals and Materials International. 2008;14(6):667-672.

3 García de Andrés C, Caballero FG, Capdevila C, Álvarez FL. Application of Dilatometric Analysis to the Study of Solid-Solid Phase Transformations in Steels. Materials Characterization. 2002;48:101-111.

4 Martins CA, Poliak E, Godefroid L. B., Fonstein N. Determining the Conditions for Dynamic Recrystallization in Hot Deformation of C-Mn-V Steels and the Effects of $\mathrm{Cr}$ and Mo Additions. ISIJ International. 2014;54(1):227-234.

5 Asahi H. Effects of Mo Addition and Austenitizing Temperature on Hardenability of Low Alloy B-Added Steels. ISIJ International. 2002;42(10):1150-1155.

6 Mohrbacher H. Principal Effect of Mo in HSLA Steels and Cross Effects with Microalloying Elements. In: Proceedings of the International Seminar on Applications of Mo in Steels; 2010; Beijing, China. China; 2010. p.75-96.

7 Han F, Hwang B, Suh,DW, et al. Effect of Molybdenum and Chromium on Hardenability of Low-Carbon Boron-Added Steels. Metals and Materials International. 2008;14(6):667-672.

8 Dong H, Dangsheng M, Yuping L, et al. Effects and Applications of Mo in Alloy Steels. In: Proceedings of the International Seminar on Applications of Mo in Steels; 2010; Beijing, China. China; 2010. p.2-13.

9 Shinjun S, Quilong W. The Role and Applications of Molybdenum Element in Low Alloy Steels. In: Proceedings of the International Seminar on Applications of Mo in Steels; 2010; Beijing, China. China; 2010. p. 61-74.

10 Mohrbacher $\mathrm{H}$. Combined effects of $\mathrm{Nb}$ and $\mathrm{B}$ microalloying in molybdenum based ultra low carbon bainitic (ULCB) steels. Belgium; 2004.

11 Mukherjeea S, Timokhinaa IB, Zhub C, et al. Effect of Thermo-mechanical Processing on Precipitate Formation in Next Generation HSLA Steel. Report from University of Sydney, NSW, Austrália; 2010.

12 Hutchinson B. Different Roles for Vanadium as a Microalloying Element in Structural Steels. Corrosion and Metals Research Institute; 2005.

13 Mamdouh E, Ahmed AE, Saeed G, et al. Effect of Microalloying Additions on the Microstructure and Mechanical Properties of Low Carbon Steel. German University in Cairo "GUC"; 2010. p.5.

14 Lagneborg R, Siwecki T, Zajac S, Hutchinson B. The Role of Vanadium in Microalloyed Steels. Swedish Institute for Metals Research; 1999. p.86.

15 Rees GI, Perdrix J, Maurickx T, Bhadeshia HKDH. The Effect of Niobium in Solid Solution on the Transformation Kinetics of Bainite. Material Science and Engineering. 1995;A194:179-186.

16 Manohar PA, Chandra T. Continuous Cooling Transformation Behavior of High Strength Microalloyed Steels for Pipeline Applications. ISIJ International. 1998;38(7):766-774.

17 Schastlivtsev V, Mirzayev DA, Karzunov SE, et al. New Concepts of Bainitic and Martensitic Transformations in Steels Based on Multistep Austenite-Ferrite Transformation. ISIJ International. 1995;35(8):955-961.

\footnotetext{
* Contribuição técnica ao $69^{\circ}$ Congresso Anual da ABM - Internacional e ao 14ํㅡㄹ ENEMET - Encontro Nacional de Estudantes de Engenharia Metalúrgica, de Materiais e de Minas, 21 a 25 de julho de 2014, São Paulo, SP, Brasil.
} 
18 Qu J, Shan Y, Zhao M, et al. Influence of Hot Deformation and Cooling Conditions on the Microstructures of Low Carbon Microalloyed Steels. Journal of Material Science and Technology. 2001;17:135-138.

19 Ansell GS, Donachie SJ, Messler Jr. RW. The effect of Quench Rate on the Martensitic Transformation in Fe-C Alloys. Metallurgical Transaction. 1971;2:2443-2449.

20 Krauss G, Marder AR. The Morphology of Martensite in Iron Alloys. Metallurgical Transactions. 1971;2:2343-2357.

* Contribuição técnica ao 69ำ Congresso Anual da ABM - Internacional e ao 14ํㅡㄹ ENEMET - Encontro Nacional de Estudantes de Engenharia Metalúrgica, de Materiais e de Minas, 21 a 25 de julho de 2014, São Paulo, SP, Brasil. 